\title{
Relationship between Tumor Mutational Burden, PD-L1, Patient Characteristics, and Response to Immune Checkpoint Inhibitors in Head and Neck Squamous Cell Carcinoma
}

\author{
Kimberly M. Burcher ${ }^{1,+}{ }^{,}$Jeffrey W. Lantz ${ }^{1,+}$, Elena Gavrila ${ }^{1}$, Arianne Abreu ${ }^{2}$, Jack T. Burcher ${ }^{3}$, \\ Andrew T. Faucheux ${ }^{1}$, Amy Xie ${ }^{1}$, Clayton Jackson ${ }^{1}$, Alexander H. Song ${ }^{1}$, Ryan T. Hughes ${ }^{1}$, Thomas Lycan, Jr. ${ }^{1}$, \\ Paul M. Bunch ${ }^{1}$, Cristina M. Furdui ${ }^{1}$, Umit Topaloglu ${ }^{1}$ [D, Ralph B. D’Agostino, Jr. ${ }^{1}$, Wei Zhang ${ }^{1}$ \\ and Mercedes Porosnicu ${ }^{1, *}$
}

1 Wake Forest Baptist Medical Center, Winston-Salem, NC 27157, USA; kburcher@wakehealth.edu (K.M.B.); jwlantz@wakehealth.edu (J.W.L.); egavrila@wakehealth.edu (E.G.); afaucheu@wakehealth.edu (A.T.F.); axie@wakehealth.edu (A.X.); cwjackso@wakehealth.edu (C.J.); asong@wakehealth.edu (A.H.S.); ryhughes@wakehealth.edu (R.T.H.); tlycan@wakehealth.edu (T.L.J.); pbunch@wakehealth.edu (P.M.B.); cfurdui@wakehealth.edu (C.M.F.); Umit.Topaloglu@wakehealth.edu (U.T.); rdagosti@wakehealth.edu (R.B.D.J.); wezhang@wakehealth.edu (W.Z.)

2 Lewisgale Medical Center, Salem, VA 24153, USA; a_abreu0419@email.campbell.edu

check for updates

Citation: Burcher, K.M.; Lantz, J.W.; Gavrila, E.; Abreu, A.; Burcher, J.T.; Faucheux, A.T.; Xie, A.; Jackson, C.; Song, A.H.; Hughes, R.T.; et al. Relationship between Tumor Mutational Burden, PD-L1, Patient Characteristics, and Response to Immune Checkpoint Inhibitors in Head and Neck Squamous Cell Carcinoma. Cancers 2021, 13, 5733. https://doi.org/10.3390/ cancers 13225733

Academic Editors: Nandini Dey and Pradip De

Received: 24 September 2021

Accepted: 29 October 2021

Published: 16 November 2021

Publisher's Note: MDPI stays neutral with regard to jurisdictional claims in published maps and institutional affiliations.

Copyright: (c) 2021 by the authors. Licensee MDPI, Basel, Switzerland. This article is an open access article distributed under the terms and conditions of the Creative Commons Attribution (CC BY) license (https:/ / creativecommons.org/licenses/by/ $4.0 /)$.
3 Lake Erie College of Medicine, Bradenton, FL 34211, USA; jburcher83447@med.lecom.edu

Correspondence: mporosni@wakehealth.edu

+ These authors equally contributed to the study.

Simple Summary: Immunotherapy has prompted a dramatic change in the management of head and neck squamous cell carcinoma (HNSCC), but the percentage of patients benefiting from treatment is limited to $20 \%$ or less. The application of precision oncology to HNSCC introduces the potential for the emergence of biomarkers that may predict a response to immunotherapy and assist with the selection of patients that may benefit from treatment with an immune checkpoint inhibitors. In this retrospective study, the results of tumor mutational burden and programmed death ligand1 measurements from HNSCC tumors were evaluated independently for their associations with demographics, risk factors, disease characteristics, survival, and response to ICI. Results of this study are expected to assist in laying the groundwork for creating a framework in which PD-L1 and TMB coexist with other variables to predict response to ICI on an individual level.

Abstract: Failure to predict response to immunotherapy (IO) limited its benefit in the treatment of head and neck squamous cell cancer (HNSCC) to $20 \%$ of patients or less. Biomarkers including tumor mutational burden (TMB) and programmed death ligand-1 (PD-L1) were evaluated as predictors of response to $\mathrm{IO}$, but the results are inconsistent and with a lack of standardization of their methods. In this retrospective study, TMB and PD-L1 were measured by commercially available methodologies and were correlated to demographics, outcome, and response to PD-1 inhibitors. No correlation was found between TMB and PD-L1 levels. High TMB was associated with smoking and laryngeal primaries. PD-L1 was significantly higher in African Americans, patients with earlier stage tumors, nonsmokers, and nonethanol drinkers. Patients with high TMB fared better in univariate and multivariate survival analysis. No correlation was found between PD-L1 expression and prognosis. There was a statistically significant association between PFS and response to IO and TMB. There was no association between response to ICI and PD-L1 in this study, possibly affected by variations in the reporting method. Further studies are needed to characterize the biomarkers for IO in HNSCC, and this study supports further research into the advancement of TMB in prospective studies.

Keywords: HNSCC; TMB; immunotherapy; immune checkpoint inhibitors; PD-L1 


\section{Introduction}

Prognoses in oncology have dramatically improved with the development of immunotherapy (IO), particularly with the advent of immune checkpoint inhibitors (ICI). Antibodies to programmed death-1 (PD-1) receptor and the programmed death ligand-1 (PD-L1) are specific kinds of ICI which function by inhibiting the binding of the programmed death-1 (PD-1) receptor to PD-L1, thus allowing tumor cells to be recognized as "other" and eliminated by a patient's immune system (Figure 1) [1]. Emerging data indicate patients with HNSCC are likely to benefit from breakthroughs in ICI, presumably due to the high levels of circulating immune cells and high levels of neoantigens within these tumors (Figure 1) [2]. Clinical trials evaluating the response to ICIs in recurrent unresectable and metastatic HNSCC have shown significant improvement in overall survival (OS) when PD-1 inhibitors are utilized alone or in combination with chemotherapy [3-7].

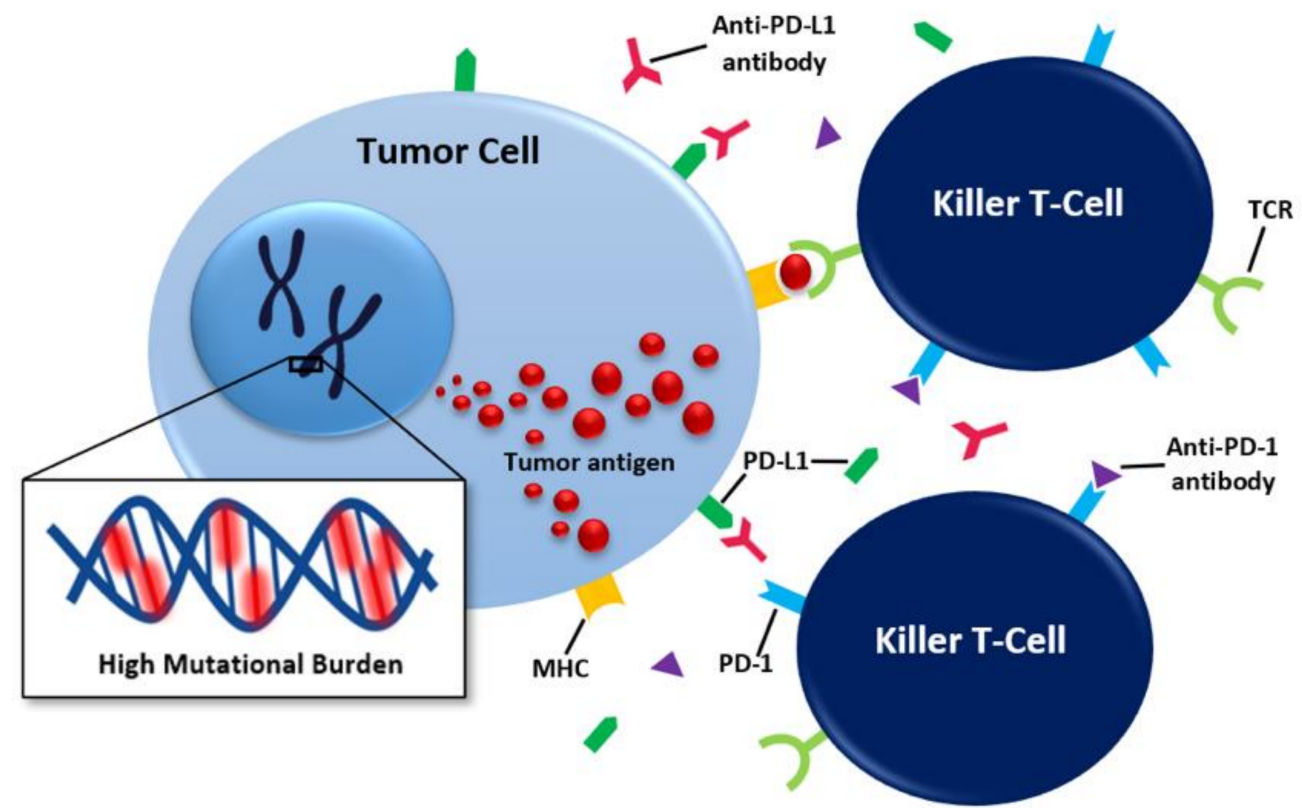

Figure 1. A brief review of the mechanism of action of PD-1/PD-L1 ICIs. Abbreviations: MHC, major histocompatibility complex; PD-1, programmed death-1; PD-L1, programmed death ligand-1; and TCR, T-cell receptor.

Despite these promising developments, fewer than $20 \%$ of HNSCC patients respond to treatment with ICI within the FDA approved setting, with the majority of patients displaying primary resistance [8]. With current clinical trials seeking to advance PD-1 ICI to curative settings, improved patient selection as a means of increasing the percentage of responders is critical, urging the development of reliable biomarkers predictive of the response to ICI. Many immune biomarkers including PD-L1 expression, tumor mutational burden (TMB), tumor immune cell infiltration, circulating immune cells, HPV, changes within the microbiome, and certain risk factors such as smoking have been suggested as predictors of HNSCC response to ICI, but the data remain in its infancy, without causative association and often with conflicting results within the literature [9]. Furthermore, there remains a limited understanding of how responses to ICI, prognosis, and each of the proposed biomarkers may be impacted by environmental factors or individual patient characteristics.

As a logical biomarker for the prediction of response to anti-PD1/PD-L1 agents, PD-L1 expression remains the only biomarker studied in prospective clinical trials in HNSCC. Studies place HNSCC amongst the malignancies with the highest frequency of PD-L1 positivity, defined as PD-L1 expression $\geq 1 \%$ when measured by either tumor proportion score (TPS) or combined positive score (CPS). It has been estimated that between $57 \%$ and $82 \%$ of HNSCC patients are PD-L1 positive $[5,10,11]$. PD-L1 expression is often described as an inexact measurement of response to ICI with levels broadly correlating to response 
rates; however, it is well known that some PD-L1 negative patients do respond to ICI, and some patients with high PD-L1 levels do not [12].

Given the inconsistencies in results produced by the utilization of PD-L1 to predict response to ICI, many alternative biomarkers have been suggested. The chief prospect amongst these alternatives is TMB, a measure of the total number of coding mutations in a tumor's genome and reported in number of mutations per mega base (mut/Mb) of DNA sequenced [13]. In theory, higher TMB conveys a higher expression of tumor neoantigens, which elicit an increased antitumor immune response, conferring greater sensitivity to ICI (Figure 1) [9]. This has been proven to be the case in many malignancies, and at times, TMB has been reported to outperform PD-L1 in prediction of response; however, the data are incomplete and occasionally contradictory, and thus no consensus has been reached regarding widespread clinical use [13-25]. Although TMB has been approved for the selection of patients with cancer for IO treatment independent of tumor type [26], few data are available in HNSCC. Additionally, few studies correlate TMB to demographics or survival of HNSCC patients, and the results of studies that do attempt these correlations remain inconsistent $[26,27]$. The predictive power of TMB in regard to response to ICI in HNSCC also has yet to be defined. To date, there are no prospective studies regarding the use of tissue TMB as a biomarker to predict response to ICI, but retrospective analyses have successfully correlated high TMB with response to ICIs in HNSCC [9,24,27-29]. Similarly, the emerging role of circulating/blood TMB remains undefined, but preliminary results are promising [30,31].

Inconsistency in results is common in the literature regarding biomarkers such as PDL1 and TMB. This is not exclusive to HNSCC. The variation in the assays used to measure these variables and reporting appear to be important contributors. Standardization efforts are ahead for PD-L1, with FDA approval of PD-L1 IHC 22C3 pharmDx reported as CPS, as a companion diagnostic for pembrolizumab treatment in HNSCC. Validation in clinical research and practice is ongoing. No such efforts have been undertaken for TMB, which has not yet been evaluated in prospective setting in HNSCC.

Joining the effort to lay a groundwork for the use of PD-L1 and TMB biomarkers to guide protocols regarding the use of PD-L1 ICI in HNSCC, this retrospective study correlates the level of expression of these biomarkers with demographic and outcome data in a dedicated HNSCC population. Additionally, the investigators correlate PD-L1 and TMB expression with response to PD-L1 ICI in the cohort of treated HNSCC patients. The primary objective of this study is to investigate the feasibility of continued pursuits of PD-L1 and TMB in prospective clinical trials in which ICI would be used to treat HNSCC. Importantly, the commercially available methodologies for measurement of the biomarkers were utilized.

\section{Materials and Methods}

This is a single-institution retrospective review of adult patients with HNSCC treated at the Wake Forest Baptist Comprehensive Cancer Center between August 2014 and October 2020 who had tumor tissue submitted for next-generation sequencing (NGS) and/or PD-L1 testing. The Wake Forest School of Medicine Institutional Review Board (IRB00057787) reviewed this study and granted approval. HNSCC patients were required to have had a valid TMB or PD-L1 test to be included in this study. Patients with cutaneous SCC or salivary gland cancers were excluded.

TMB was measured via FoundationOne (F1) testing (Foundation Medicine, Cambridge MA, USA) (F1). PD-L1 was analyzed by the standard, FDA-approved, immunohistochemistry 22C3 pharmDx kit, performed commercially by F1 and, in a small number of patients, by Mayo Clinic laboratories. PD-L1 expression was reported as a tumor proportion score (TPS) until 2019 and by the combined positive score (CPS) thereafter. PD-L1 was analyzed both as a 3-tiered and 2-tiered variable. The 3-tiered PD-L1 variable (3tPD-L1) was divided into categories similar to those in Keynote-048 and consisted of three groups: those with a PD-L1 of 0 (3tPD-L1-(0)), those with a PD-L1 between 1 and 19 (3tPD-L1-(1-19)) and 
those with PD-L1 greater than or equal to 20 (3tPD-L1-(20+)). Due to the low number of patients in the 3tPD-L1-(0) group, PD-L1 was also analyzed as a 2-tiered variable (2tPD-L1). In the 2tPD-L1, the 3tPD-L1-(0) and 3tPD-L1-(1-19) were grouped together and referred to as 2 tPD-L1- $(<20)$ and was compared to a group of patients with PD-L1 values greater than or equal to 20 referred to as 2tPD-L1-(20+). TMB was likewise initially divided into three categories (3-tiered-TMB) with low scores (TMB less than 6 mut/Mb), intermediate scores (TMB greater than or equal to 6 but less than 20 mut/Mb), and high scores group (TMB greater or equal to 20 mut/Mb) as recommended by F1, but, due to poor distribution across the sample population, no analysis was performed for the 3-tiered variable. Similar to PD-L1, TMB was recategorized into a 2-tiered variable, with those with TMB scored less than $6 \mathrm{mut} / \mathrm{mb}$ in the TMB- $(<6)$ category and those with TMB of 6 or greater in the TMB-(6+) category. In instances in which PD-L1 or TMB tests were repeated, the highest resulted number was reported.

Demographic data and patient characteristics were obtained from the electronic medical record and included age (greater or less than 60 years old), sex, disease stage at diagnosis per AJCC 8th edition, HPV by PCR or p16 status, smoking status (grouped as never-smokers vs. ever-smokers, where ever-smokers were defined as former or current smokers), alcohol use, tumor subsite (oral cavity, oropharynx, larynx, hypopharynx, nasopharynx, paranasal sinuses, or unknown primary), and treatment received before tumor tissue collection (chemotherapy, radiotherapy, or both).

Outcome measures included OS measured from the time of diagnosis and from the time of tumor tissue collection. Survival at 1 and 2 years measured from the date of tumor tissue collection, survival at the end of the study, and extent/burden of disease at last visit were also included in outcome data. It should be noted that for all calculations in which the extent of disease was measured, three categories were considered. These were defined as "no evidence of disease", "localized disease", and "metastatic disease". Multivariate analysis was performed for both TMB and PD-L1 groups separately.

Treatment response was measured by CT or MRI and categorized according to RECIST v1.1. Patients with complete response (CR), partial response (PR), or stable disease (SD) for at least 6 months as best overall response (BOR) were grouped in a category called "responders". Patients who progressed through the treatment (PD) without achieving a response as above were called "nonresponders". Progression-free survival (PFS) was measured from the first day of treatment with ICI to the day of confirmed tumor progression, or to the day of death if the patient died before tumor progression was documented, or until the last visit if there was no tumor progression.

\section{Statistical Analysis}

Descriptive statistics were calculated for all variables. These included means and standard deviations for continuous measures and counts and percentages for categorical measures. TMB and PD-L1 levels were portioned into tiers as described above (TMB into 2 tiers and PD-L1 into 2 or 3 tiers). We then examined the association between categorical variables and the TMB/PD-L1 groupings using Fisher's exact test (for binary variables) and chi-square test for categorical variables with 3 or more levels. Continuous variables were compared across TMB/PD-L1 groups using $t$-tests (for 2 tier groups) and one-way analysis of variance models for 3tPD-L1. Time-to-event data were examined in two ways: one examining time from diagnosis until event (i.e., death) and the second examining time from testing until event. Kaplan-Meier curves were generated for examining survival distributions both overall and by TMB or PD-L1 groups. Log-rank tests were used to compare groups. Next, Cox proportional hazards regression models were fit to examine the relationship of TMB or PD-L1 groups with survival after adjusting for patient level characteristics including age, tobacco use, tumor site, stage at diagnosis, and prior treatment with combined chemoradiation therapy. Next, we evaluated treatment response to immunotherapy as BOR and PFS. Fisher's exact tests and chi-square tests (as described above) were used to determine whether PD-L1 and TMB categories were associated with 
the BOR treatment categories. We compared average PFS days between responders and nonresponders using a 2-sample t-test. Next, we compared PFS days by PD-L1 and TMB categories as described above using 1-way ANOVA models and PD-L1 and TMB levels (as continuous values) using Pearson correlations. Hazard ratios and corresponding 95\% confidence intervals were estimated from these proportional hazard regression models. In all analyses, an alpha level of 0.05 was used to determine the significance of data. Statistical analysis system (SAS) 9.4 was used to perform all analyses in this study.

\section{Results}

\subsection{Patient Characteristics}

In total, 139 patients met inclusion criteria for this study. Of these, 128 patients had TMB results, 95 patients had PD-L1, and 92 patients had results for both metrics. The demographic and disease characteristics of the patients included in this analysis are available for review in Table 1. Age, race, and gender in this study are congruent with a standard population of patients with HNSCC.

Table 1. Characteristics of all patients included in the study.

\begin{tabular}{|c|c|c|c|c|c|}
\hline \multirow[t]{2}{*}{ Characteristics } & $\begin{array}{c}\text { TMB } \\
\text { Patients }\end{array}$ & $\begin{array}{c}\text { PD-L1 } \\
\text { Patients }\end{array}$ & \multirow[t]{2}{*}{ Characteristics } & $\begin{array}{c}\text { TMB } \\
\text { Patients }\end{array}$ & $\begin{array}{c}\text { PD-L1 } \\
\text { Patients }\end{array}$ \\
\hline & No. $(\%)$ & No. (\%) & & No. (\%) & No. (\%) \\
\hline Age at Diagnosis (Years) & & & Primary Tumor Location & & \\
\hline Median & 60 & 61 & Nasopharynx & $8(6.2)$ & $7(7.4)$ \\
\hline$\geq 60$ & $64(50)$ & $50(52.6)$ & Oropharynx & $50(39.1)$ & $37(38.9)$ \\
\hline \multirow[t]{2}{*}{$<60$} & $64(50)$ & $45(47.4)$ & Oral Cavity & $33(25.8)$ & $23(24.2)$ \\
\hline & & & Hypopharynx & $7(5.5)$ & $5(5.3)$ \\
\hline Gender & & & Larynx & $22(17.2)$ & $16(16.8)$ \\
\hline Male & $89(69.5)$ & $68(71.6)$ & Sino-Nasal & $5(3.9)$ & $4(4.2)$ \\
\hline Female & $39(30.4)$ & $27(28.4)$ & Unknown & $3(2.3)$ & $3(3.2)$ \\
\hline Race & & & Disease Stage at Time of & & \\
\hline Caucasian & $108(84.4)$ & $79(83.2)$ & Diagnosis & & \\
\hline African American & $13(10.2)$ & $13(13.7)$ & & & \\
\hline \multirow[t]{2}{*}{ Other } & $7(5.4)$ & $3(3.1)$ & Cancer Stage & & \\
\hline & & & I & $19(14.8)$ & $17(17.9)$ \\
\hline ETOH Status & & & II & $21(16.4)$ & $18(18.9)$ \\
\hline Never & $63(49.2)$ & $46(48.4)$ & III & $29(22.7)$ & $15(15.8)$ \\
\hline Former & $28(21.9)$ & $22(23.2)$ & IV & $59(46.1)$ & $45(47.4)$ \\
\hline \multirow[t]{2}{*}{ Active } & $37(28.9$ & $27(28.4)$ & & & \\
\hline & & & Cancer Stage IV & & \\
\hline Smoking Status & & & IVA & $39(66.1)$ & $29(64.4)$ \\
\hline Never & $37(28.9)$ & $29(30.5)$ & IVB & $14(23.7)$ & $11(24.4)$ \\
\hline Former & $39(30.5)$ & $30(31.6)$ & IVC & $6(10.2)$ & $5(11.2)$ \\
\hline \multirow[t]{2}{*}{ Active } & $52(40.6)$ & $36(37.9)$ & & & \\
\hline & & & N Stage & & \\
\hline HPV and/or p16 & & & No & $37(28.9)$ & $27(28.4)$ \\
\hline Negative & $56(43.8)$ & $43(45.3)$ & N1 & $29(22.7)$ & $20(21.1)$ \\
\hline Positive & $40(31.2)$ & $29(30.5)$ & N2 & 49 (38.3) & 37 (38.9) \\
\hline Not Tested & $32(25)$ & $23(24.2)$ & N3 & $13(10.1)$ & 11 (11.6) \\
\hline BMI & & & Tissue & & \\
\hline & & & Source & & \\
\hline$<18.5$ & $19(14.7)$ & 11 (11.9) & Primary Tumor & $83(65.4)$ & $55(60.4)$ \\
\hline $18.5-24.9$ & $42(32.6)$ & $33(35.9)$ & Regional Node & $11(8.7)$ & $10(11.0)$ \\
\hline $25-29.9$ & $39(30.2)$ & $30(32.6)$ & Metastatic Lesion & $11(8.7)$ & $9(9.9)$ \\
\hline$\geq 30$ & $29(22.5)$ & 18 (19.6) & Recurrence & $22(17.3)$ & 17 (18.7) \\
\hline
\end{tabular}

Abbreviations: BMI, body mass index; HPV, human papilloma virus; PD-L1, programmed death ligand-1; TMB, tumor mutational burden. 


\subsection{Prevalence of PD-L1 and TMB within the Study Population and Correlation between the} Two Variables

Of the 95 patients with recorded measurements of PD-L1 expression, 80 patients $(84 \%)$ had results from testing performed by $\mathrm{F} 1$, and 15 patients (16\%) had results from testing performed by the Mayo Clinic Laboratory. PD-L1 was measured by TPS in 52 patients (55\%) and by CPS in the remaining 43 patients (45\%). The mean PD-L1 score was $26.41 \%$ (standard deviation 33.78 ). The median score was $10 \%$. Twelve patients $(13 \%)$ had 3tPD-L1-(0). In addition, 46 patients (48\%) had 3tPD-L1-(1-19), and 37 patients (39\%) had 3tPD-L1-(20+) (Figure 2A). The 2tPD-L1 re-distribution resulted in 58 patients $(61 \%)$ in the 2tPD-L1- $(<20)$ group and 37 patients $(39 \%)$ in the 2tPD-L1- $(20+)$ group.

(A)

PD-L1

(B)

TMB
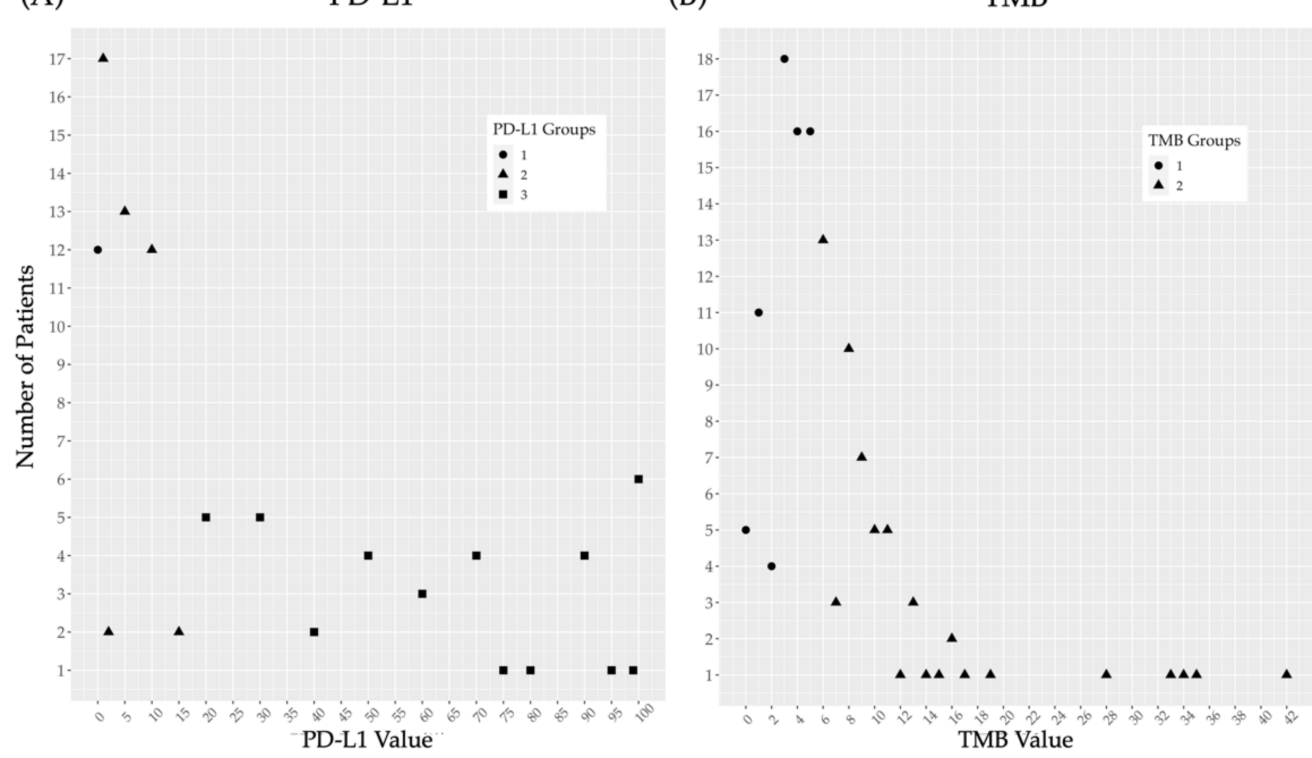

(C)

PD-L1 vs. TMB

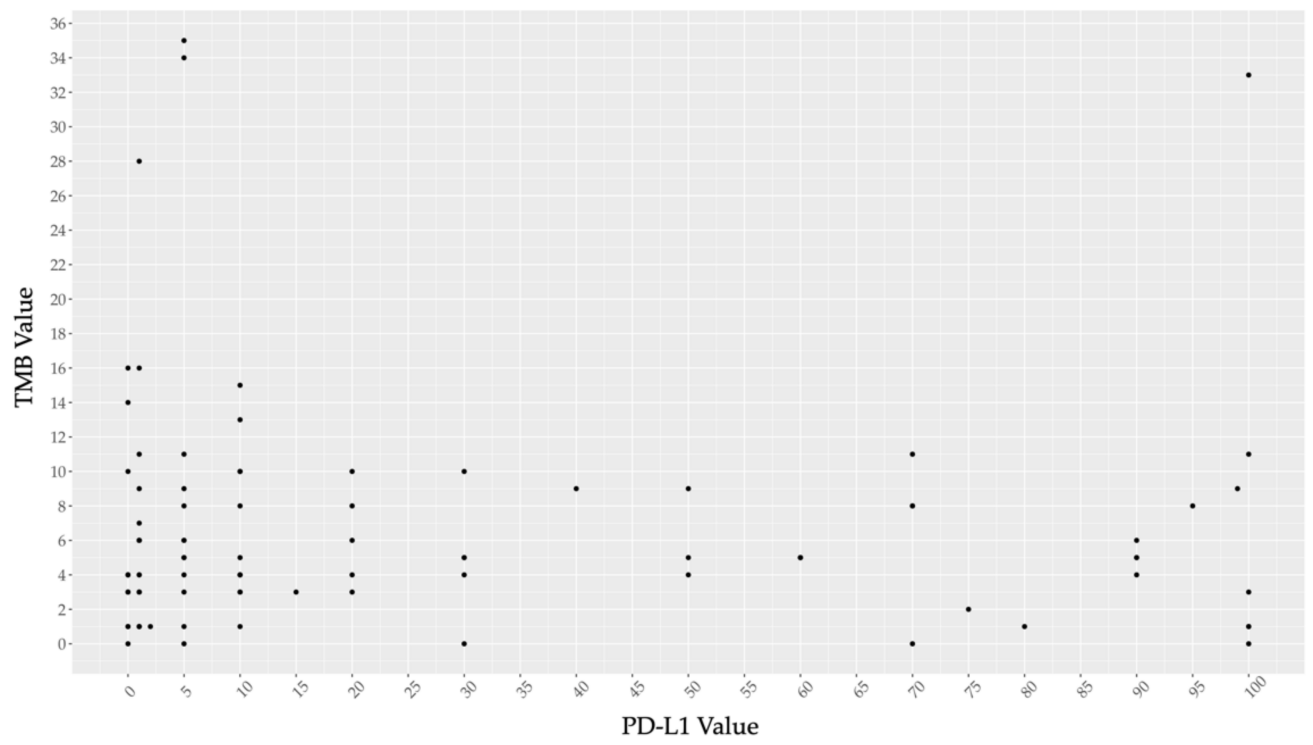

Figure 2. Distribution of continuous PD-L1 and continuous TMB and scatter plot demonstrating the relationship between each. (A) Distribution of PD-L1 across 3tPD-L1-(0) (group 1, circles), 3tPD-L1-(1-19) (group 2, triangles) and 3tPD-L1-(20+) (group 3, squares); (B) Distribution of TMB across TMB-(<6) (group 1, circles) and TMB-(6+) (group 2, triangles); (C) Scatter plot demonstrating the failure of PD-L1 to correlate to TMB. Abbreviations: PD-L1, programmed death ligand-1; and TMB, tumor mutational burden. 
The mean TMB of the 128 patients included in TMB analysis was 6.98 mut/Mb (standard deviation 6.85), and the median was 5.0 mut/Mb. The TMB- $(<6)$ category consisted of 70 patients $(55 \%)$, and 58 patients $(45 \%)$ were in the TMB- $(6+)$ category (Figure $2 \mathrm{~B})$.

A total of 92 patients had both PD-L1 and TMB testing results available. Of these, 12 patients (13\%) were in the 3tPD-L1-(0) category, 44 patients $(48 \%)$ were in the $3 \mathrm{tPD}$ L1-(1-19) category, and 35 patients (39\%) were in the 3tPD-L1-(20+) category. In addition, 38 patients $(42 \%)$ were in the TMB- $(6+)$ category, and 53 patients $(58 \%)$ were in the TMB$(<6)$ category. There were no statistically significant correlations identified between PD-L1 expression and TMB as categorical variables $(p=0.84)$ (Figure $2 \mathrm{C}$ ).

\subsection{Correlation between PD-L1 Expression and Patient Characteristics}

African Americans had statistically significant higher PD-L1 expression than Caucasians, with $69.2 \%$ vs. $35.4 \%$ classified in the 2 tPD-L1 $\geq 20(p=0.04)$. The analysis lost statistical significance in the three-tiered variant. There were no significant correlations identified between age or gender and 3tPD-L1 or 2tPD-L1, although it was noted that patients older than 60 and women regardless of age had a tendency toward higher PD-L1 expression. There was no significant correlation between HPV status and PD-L1, both in 2tPD-L1 ( $p=0.74)$ and 3tPD-L1 ( $p=0.35)$ analysis; however, in the 3tPD-L1 analysis it was noted that there were no HPV-positive patients in the 3tPD-L1-(0) category, while 18.6\% of HPV-negative patients were 3tPD-L1-(0) (Table 2).

Table 2. Correlation of select demographic and patient specific data with 3-tiered-PD-L1 and 2-tiered TMB categories.

\begin{tabular}{|c|c|c|c|c|c|c|c|c|}
\hline & & \multicolumn{4}{|c|}{ 3tPD-L1 Analysis } & \multicolumn{3}{|c|}{ TMB Analysis } \\
\hline \multicolumn{2}{|c|}{ Variable } & \multicolumn{3}{|c|}{ No. (Row \%) } & \multirow{2}{*}{$p$} & \multicolumn{2}{|c|}{ No. (Row \%) } & \multirow{2}{*}{$p$} \\
\hline & & $\geq \mathbf{2 0}$ & $1-19$ & 0 & & $\geq 6$ & $<6$ & \\
\hline \multirow{2}{*}{ Age } & $<60$ & $14(31.1)$ & $25(55.6)$ & $6(13.3)$ & \multirow{2}{*}{0.240} & $31(48.4)$ & $33(51.6)$ & \multirow{2}{*}{0.478} \\
\hline & $\geq 60$ & $23(46.0)$ & $21(42.0)$ & $6(12.0)$ & & $27(42.2)$ & $37(57.8)$ & \\
\hline \multirow[b]{2}{*}{ Gender } & Male & $24(35.3)$ & $35(51.5)$ & $9(13.2)$ & \multirow{2}{*}{0.510} & $37(41.6)$ & $52(58.4)$ & \multirow[b]{2}{*}{0.199} \\
\hline & Female & $13(48.1)$ & $11(40.7)$ & $3(11.1)$ & & $21(53.8)$ & $18(46.2)$ & \\
\hline \multirow{2}{*}{ Race } & Caucasian & $28(35.4)$ & $41(51.9)$ & $10(12.7)$ & \multirow[b]{2}{*}{0.046} & $52(48.1)$ & $56(51.9)$ & \multirow{2}{*}{$\underline{0.087}$} \\
\hline & AA & $9(69.2)$ & $2(15.4)$ & $2(15.4)$ & & $3(23.1)$ & $10(76.9)$ & \\
\hline \multirow{2}{*}{ HPV } & + & $11(38.0)$ & $18(62.1)$ & $0(0.0)$ & \multirow{2}{*}{0.354} & $16(40.0)$ & $24(60.0)$ & \multirow{2}{*}{0.804} \\
\hline & - & $18(41.9)$ & 17 (39.5) & $8(18.6)$ & & $21(37.5)$ & $35(62.5)$ & \\
\hline \multirow{3}{*}{$\begin{array}{l}\text { Smoking } \\
\text { History }\end{array}$} & Current & $12(33.3)$ & $17(47.2)$ & 7 (19.4) & \multirow{3}{*}{0.044} & $31(59.6)$ & $21(40.4)$ & \multirow{3}{*}{0.029} \\
\hline & Former & $10(33.3)$ & $16(53.3)$ & $4(13.3)$ & & $13(33.3)$ & $26(66.7)$ & \\
\hline & Never & $15(51.7)$ & $13(44.8)$ & $1(3.4)$ & & $14(37.8)$ & $23(62.2)$ & \\
\hline \multirow{3}{*}{$\begin{array}{l}\text { Alcohol } \\
\text { History }\end{array}$} & Current & $7(25.9)$ & $15(55.6)$ & $5(18.5)$ & \multirow{3}{*}{0.012} & $18(48.6)$ & $19(51.4)$ & \multirow{3}{*}{0.326} \\
\hline & Former & $6(27.3)$ & $12(54.5)$ & 4 (18.2) & & $15(53.6)$ & $13(46.4)$ & \\
\hline & Never & $24(52.2)$ & $19(41.3)$ & $3(6.5)$ & & $25(39.7)$ & $38(60.3)$ & \\
\hline \multirow{2}{*}{ BMI } & $\geq 30$ & $10(55.5)$ & $7(38.9)$ & $1(5.6)$ & \multirow{2}{*}{$\underline{0.072}$} & $9(31.0)$ & $20(69.0)$ & \multirow{2}{*}{$\underline{0.071}$} \\
\hline & $<30$ & $25(33.8)$ & $37(50.0)$ & $12(16.2)$ & & $50(50.0)$ & $50(50.0)$ & \\
\hline \multirow{2}{*}{$\begin{array}{l}\text { Previous } \\
\text { CRT }\end{array}$} & + & $15(41.7)$ & $16(44.4)$ & $5(13.9)$ & \multirow[b]{2}{*}{0.868} & $23(51.1)$ & $22(48.9)$ & \multirow[b]{2}{*}{0.331} \\
\hline & - & $22(37.3)$ & $30(50.8)$ & 7 (11.9) & & $35(42.2)$ & $48(57.8)$ & \\
\hline & OC & $10(43.5)$ & $11(47.8)$ & $2(8.7)$ & & $16(48.5)$ & $17(51.5)$ & \\
\hline & Other & $21(36.2)$ & $28(48.3)$ & $9(15.5)$ & 0.400 & 35 (44.3) & $44(55.7)$ & 0.685 \\
\hline Tumor & Laryngeal & $6(37.5)$ & $8(50)$ & $2(12.5)$ & & $16(72.7)$ & $6(27.3)$ & \\
\hline Location & OTT & 25 (38.5) & $31(47.7)$ & $9(13.8)$ & 0.984 & $35(38.9)$ & 55 (61.1) & 0.004 \\
\hline & OP & $15(40.5)$ & $16(43.2)$ & $6(16.2)$ & & $15(30.0)$ & $35(70.0)$ & \\
\hline & Other & $16(36.4)$ & $23(52.3)$ & $5(11.4)$ & 0.965 & $36(58.1)$ & $26(41.9)$ & .003 \\
\hline
\end{tabular}


Table 2. Cont.

\begin{tabular}{|c|c|c|c|c|c|c|c|c|}
\hline & & \multicolumn{4}{|c|}{ 3tPD-L1 Analysis } & \multicolumn{3}{|c|}{ TMB Analysis } \\
\hline \multicolumn{2}{|c|}{ Variable } & \multicolumn{3}{|c|}{ No. (Row \%) } & \multirow{2}{*}{$p$} & \multicolumn{2}{|c|}{ No. (Row \%) } & \multirow{2}{*}{$p$} \\
\hline & & $\geq 20$ & $1-19$ & 0 & & $\geq 6$ & $<6$ & \\
\hline \multirow{15}{*}{$\begin{array}{c}\text { Stage at } \\
\text { Diagnosis }\end{array}$} & I & $10(58.8)$ & $7(41.2)$ & $0(0.0)$ & \multirow{4}{*}{0.035} & $7(36.8)$ & $12(63.2)$ & \multirow{4}{*}{0.522} \\
\hline & II & $7(38.9)$ & $11(61.1)$ & $0(0.0)$ & & $11(52.4)$ & $10(45.5)$ & \\
\hline & III & $4(26.7)$ & $6(40.0)$ & $5(33.3)$ & & $11(37.9)$ & $18(62.1)$ & \\
\hline & IV & $16(35.6)$ & $22(48.9)$ & $7(15.6)$ & & $29(49.2)$ & $30(50.8)$ & \\
\hline & IVA & $5(71.4)$ & $2(28.6)$ & $0(0.0)$ & \multirow{3}{*}{0.802} & $22(56.4)$ & $17(43.6)$ & \multirow{3}{*}{0.146} \\
\hline & IVB & $13(59.1)$ & $6(27.3)$ & 3 (13.6) & & $5(35.7)$ & $9(64.3)$ & \\
\hline & IVC & $11(68.8)$ & $3(18.8)$ & $2(12.5)$ & & $2(33.3)$ & $4(66.7)$ & \\
\hline & T0-2 & $20(46.5)$ & $23(53.5)$ & $0(0.0)$ & \multirow{2}{*}{0.008} & $23(42.6)$ & $31(57.4)$ & \multirow[b]{2}{*}{0.864} \\
\hline & T3-4 & $17(54.8)$ & $2(6.5)$ & $12(38.7)$ & & $39(52.7)$ & $35(47.3)$ & \\
\hline & No & $11(40.7)$ & $10(37.0)$ & $6(19.4)$ & \multirow{4}{*}{0.949} & $17(45.9)$ & $20(54.1)$ & \multirow{4}{*}{0.751} \\
\hline & N1 & $9(45.0)$ & $11(55.0)$ & $0(0.0)$ & & 11 (37.9) & $18(62.1)$ & \\
\hline & N2 & $13(35.1)$ & $20(54.0)$ & $4(10.8)$ & & $24(49.0)$ & $25(51.0)$ & \\
\hline & N3 & $4(36.3)$ & $5(45.5)$ & $2(18.2)$ & & $6(46.2)$ & $7(53.8)$ & \\
\hline & M0 & $34(38.2)$ & $43(48.3)$ & $12(13.5)$ & \multirow{2}{*}{0.372} & $54(45.0)$ & $66(55.0)$ & \multirow{2}{*}{0.784} \\
\hline & M1 & $3(50.0)$ & $3(50.0)$ & $0(0.0)$ & & $4(50.0)$ & $4(50.0)$ & \\
\hline
\end{tabular}

Results with $p<0.05$ are bolded in italics; results with $0.05<p<0.10$ are italicized and underlined. 3tPD-L1, 3-tiered PD-L1; AA, African American; BMI, Body Mass Index; CRT, chemoradiotherapy; HPV, human papilloma virus; OC, oral cavity; OP, oropharyngeal; OTT, other throat tumors; PD-L1, programmed death ligand-1; and TMB, tumor mutational burden.

In the evaluation of modifiable traits, current nonsmokers had a significantly higher percentage of patients in the 3tPD-L1-(20+) category when compared with current smokers $(51.7 \%$ vs. $33.3 \% ; p=0.04)$. The same significance held true when never-smokers were compared to ever-smokers (current and former smokers) $(p=0.04)$. Similarly, PD-L1 and alcohol use were associated in a statistically significant manner. More than half of never-drinkers $(52.2 \%)$ vs. only $25.9 \%$ of drinkers were within the 3tPD-L1-(20+) category $(p=0.01)$. The statistical advantage was maintained in the 2tPD-L1 analysis $(p=0.04)$. Low PD-L1 was also noted to be associated with low BMI, but this trend did not reach significance (2tPD-L1 $p=0.088$ and 3tPD-L1 $p=0.07$ ). Exposure to radiotherapy, chemotherapy, or to chemoradiotherapy before tumor tissue collection for NGS or PD-L1 testing did not correlate with either PD-L1 analysis.

There was no correlation found between 2tPD-L1 or 3tPD-L1 expression and the primary tumor site ( $p=0.36$ and $p=0.61$, respectively), and no trends were identified to influence further analysis.

A statistically significant correlation was found in the 3tPD-L1 analysis of the disease stage (I-IV) at diagnosis, with a higher percentage of patients with early-stage disease having 3tPD-L1-(20+) (58.8\% patients with stage I disease vs. 35.6\% with stage IV disease; $p=0.04)$. In particular, PD-L1 associated with tumor stage but not with nodal stage. In early tumor stages (T0-T2), 46.5\% of patients were identified with 3tPD-L1-(20+) vs. only $32.7 \%$ of patients with tumor stages T3-T4, and $0 \%$ patients with early T stage vs. $23 \%$ of patients with advanced T stages were in the 3tPD-L1-(0) group $(p<0.01)$.

\subsection{Correlation between $T M B$ and Patient Characteristics}

Due to the extremely low proportion of patients within TMB 20+ category (just five patients) in the three-tiered TMB variable (Figure 2B), no attempts were made to a threetiered TMB correlative analysis. Going forward, all references are limited to the two-tiered TMB analysis. There were no significant correlations found between the TMB and age $(p=0.48)$ or gender. There was a notably higher proportion of women in the TMB- $(6+)$ group (53.8\% vs. $41.6 \%)$, but the association was not statistically significant $(p=0.20)$. Correlation of TMB with race showed that $48.1 \%$ of Caucasians vs. only $23 \%$ of African 
Americans had a TMB- $(6+)(p=0.09)$. Of note, there was no correlation between TMB and HPV status $(p=0.80)$.

Active tobacco users were significantly more likely to be in the TMB- $(6+)$ category when compared to former and never-smokers (60\% vs. $33.3 \%$ and vs. $37.8 \% ; p=0.03)$. There was no significant association between TMB and alcohol use. Patients with a body mass index (BMI) great than 30 were more likely to have low TMB- $(<6)(69 \%$ vs. $50 \%)$, but this trend failed to meet statistical significance $(p=0.07)$. Patients with previous exposure to chemoradiotherapy (CRT) before tumor tissue collection were more likely to be in the TMB- $(6+)$ category but this trend did not reach significance $(p=0.09)$. Exposure to treatment with radiation or chemotherapy or both prior to tissue collection was not associated with TMB.

A strongly significant correlation was found between TMB and primary tumor location $(p<0.01)$. Specifically, the patients within the TMB- $(6+)$ category were significantly more likely to have cancers of the larynx in comparison to other locations $(72.7 \%$ vs. $38.9 \%$; $p<0.01)$ and significantly less likely to have cancers of the oropharynx as opposed to other locations $(29.4 \%$ vs. $70.6 \% ; p<0.01)$. The stage at the time of diagnosis (I-IV) did not correlate to TMB. Similarly, there was no correlation between T stage, N stage, or M stage and TMB.

\subsection{Prognostic Value of PD-L1 Expression}

For all patients with PD-L1 expression data, the median follow-up time for testing was 588 days from the time of diagnosis with a median survival time of 791 days (95\% CI 708 to 1199 days). Overall, 47 patients (66\%) were alive at one year from diagnosis and $13(23.2 \%)$ were alive at two years from diagnosis. Survival at one or two years was not associated with PD-L1 expression level. More than half of surviving patients (67\%) had residual disease at last visit. There was no correlation between PD-L1 groups and the extent of disease at last visit.

PD-L1 expression did not correlate to survival in 2tPD-L1 or 3tPD-L1 analysis from time of tissue collection or from time of diagnosis. In the 2tPD-L1 analysis, the median survival in the 2tPD-L1-(<20) group was 521 days (95\% CI 412-1008 days) and was not significantly different from median survival in the 2tPD-L1-(20+) group, 541 days $(95 \%$ CI $415-666$ days) $(p=0.89)$. Survival from time of diagnosis was also found to be insignificantly different between 2tPD-L1 groups where the median survival in the 2tPD-L1- $<20)$ group was 1044 days (95\% CI 711-1292 days) and the median survival in the 2tPD-L1-(20+) group was 752 days (95\% CI 504-1320 days) ( $p=0.47$ ). Differences in survival between 3tPD-L1 groups were similarly unimpressive in regard to time from tissue collection and diagnosis ( $p=0.95$ and $p=0.51$, respectively). Additional information regarding correlation between PD-L1 and outcome/survival can be found in Table 3, Table 4, and Figure 3. In an adjusted Cox proportional hazard regression model, the impact of the 3t-PD-L1 variable was not related to survival when controlled for age, smoking, nodal status, subsite, and exposure to CRT $(p=0.09)$ and related to poorer OS in the two-tiered analysis $(p=0.03)$ (Tables 3 and 4). When TMB score was added to the adjusted variables above, the 2tPD-L1 more accurately could predict survival; however, this, too, did not quite reach significance $(p=0.051)$ (Table 4).

\subsection{Prognostic Value of TMB}

For all patients with TMB score data, the median follow-up time was 616 days from the time of cancer diagnosis with a median survival from diagnosis of 521 days (95\% CI 412-1008 days). Overall, 72 patients (69.9\%) were alive at one year from diagnosis, and $25(30.86 \%)$ were alive at two years from diagnosis. At the time of the last visit, $39 \%$ of patients had no evidence of disease, $24 \%$ had recurrent or progressive locoregional disease, $12 \%$ had metastatic disease, and $25 \%$ had locoregional and metastatic disease. There was no association between disease status at last visit and TMB $(p=0.39)$. 
Table 3. Association of high PD-L1 expression and TMB with survival outcomes.

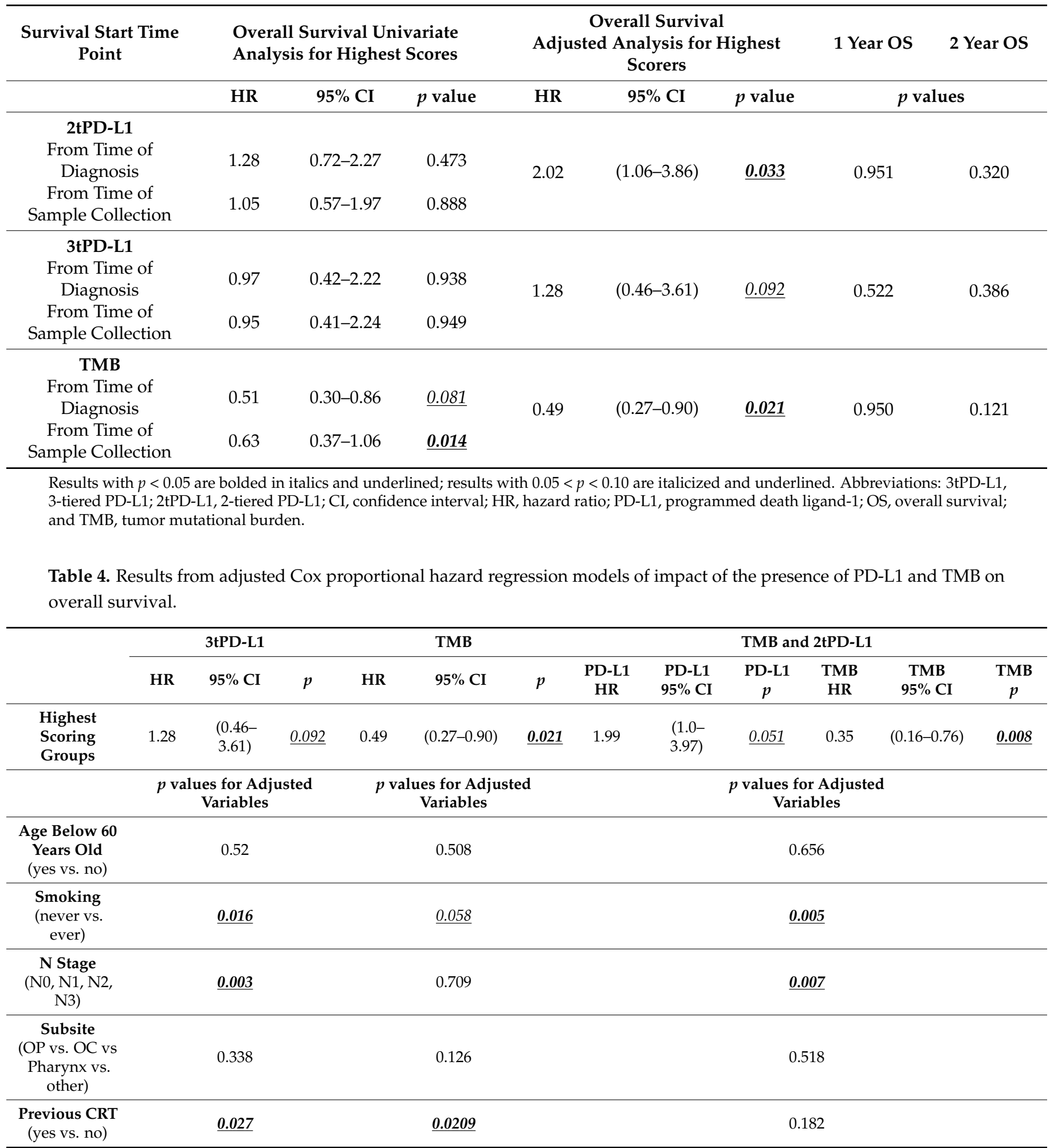

Analyses with $p<0.05$ are bolded in italics and underlined; results with $0.05<p<0.10$ are italicized and underlined; Abbreviations: 2tPD-L1, 2-tiered PD-L1; 3tPD-L1, 3-tiered PD-L1; CI, confidence interval; HR, hazard ratio; N/A, nonapplicable; CRT, combined chemotherapy and radiation therapy; PD-L1, programmed death ligand-1; and TMB, tumor mutational burden. 

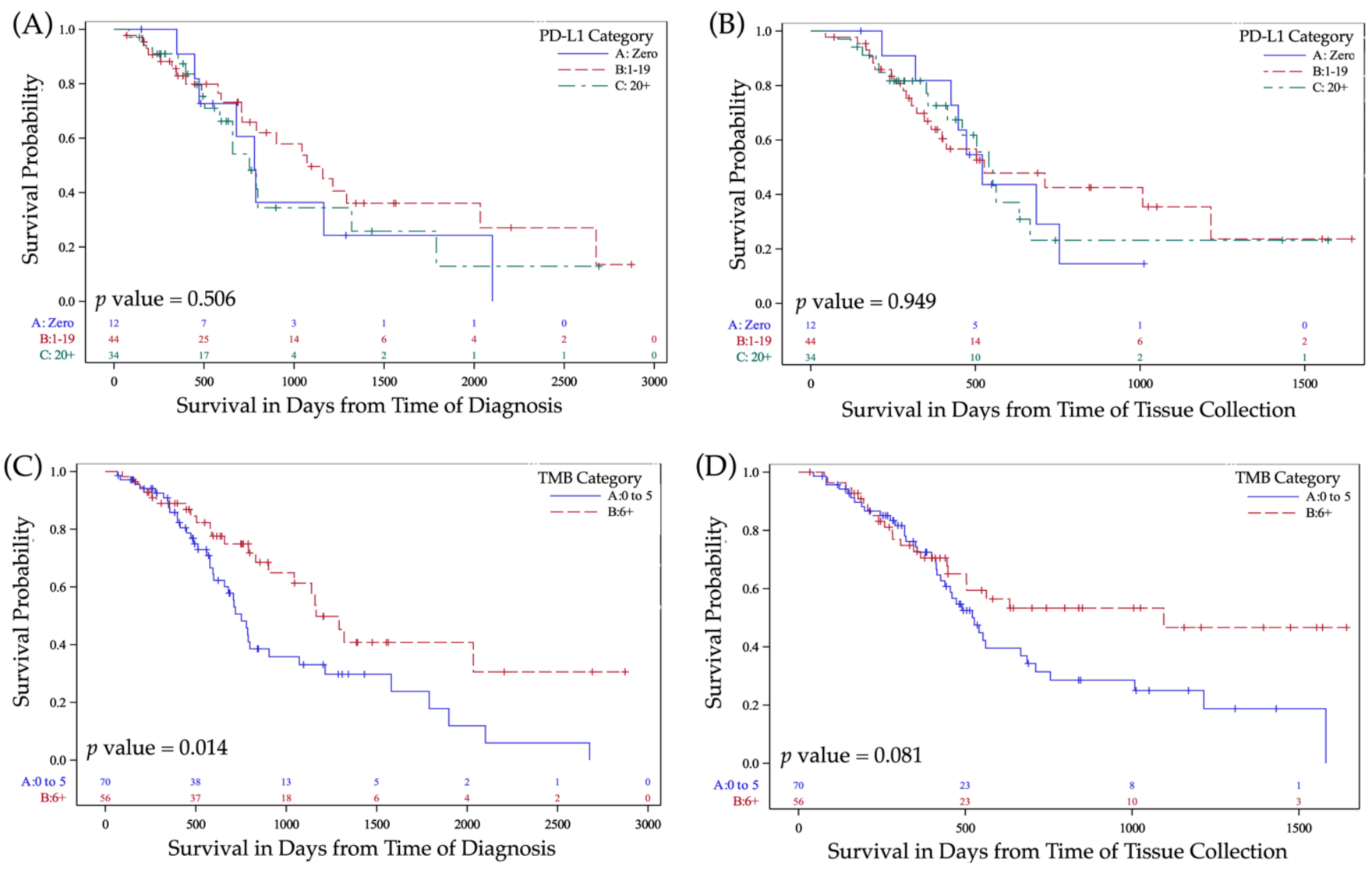

Figure 3. Kaplan -Meier curves for PD-L1 and TMB variables. (A) Survival from time of diagnosis in patients with 3tPD-L1-(20+) vs. 3tPD-L1-(1-19) vs. 3tPD-L1-(0); (B) Survival from time of tissue acquisition in patients with 3tPD-L1-(20+) vs. 3tPD-L1-(1-19) vs. 3tPD-L1-(0); (C) Survival from time of diagnosis in patients with TMB-(6+) vs. TMB-(<6); (D) Survival from time of tissue acquisition in patients with TMB-(6+) vs. TMB- $(<6)$. Abbreviations: PD-L1, programmed death ligand-1; 3tPD-L1-(20+), PD-L1 $\geq 20$; 3tPD-L1-(1-19), $0<$ PD-L1 < 20; 3tPD-L1-(0), PD-L1=0; and TMB, tumor mutational burden; TMB- $(<6)$, TMB less than 6; TMB-(6+), TMB greater than or equal to 6. Legend: Blue solid lines indicate survival curves for patients with TMB- $(<6)$ or with 3tPD-L1-(0); Red dashed lines indicate survival curves for patients with TMB-(6+) or with 3tPD-L1-(1-19); Green dashed lines indicate curves for patients with 3tPD-L1-(20+) in the 3tPD-L1 analysis. Abbreviations: PD-L1, programmed death-ligand1; and TMB, tumor mutational burden.

Generally, patients within the TMB-(6+) category fared better than those within the TMB- $(<6)$ category, but differences in survival at 1 and 2 years were not significant (Table 3$)$. Survival from the time of diagnosis was significantly better in patients within TMB-(6+) (752 days (95\% CI 599-905 days) and 1165 days (95\% CI beginning at 902 with the upper limit not yet reached, $p=0.01$ ). When considered from the time of tumor sample collection, survival was only marginally better in the TMB- $(6+)$ category $(p=0.08)$. The survival significance from the time of diagnosis was upheld in an adjusted Cox proportional hazard regression model of survival controlled for PD-L1 category, age, smoking, nodal status, subsite, and previous exposure to CRT $(p=0.02)$. This remained significant in an analysis in which 2tPD-L1 was added to the analysis $(p<0.01)$ (Table 3, Table 4 , and Figure 3).

\subsection{Treatment with PD-L1/PD-1 Inhibitors and Correlation with PD-L1 and TMB}

A total of 79 patients in this study received at least one treatment with an ICI. Treatment efficacy was able to be evaluated in 51 of these patients. Of the 28 patients that could not be evaluated, nine patients (32\%) received a planned treatment with less than three administrations of a PD-L1 inhibitor in a neoadjuvant setting and were not able to be evaluated for treatment efficacy. Nineteen patients $(68 \%)$ had treatment cessation before the initial scans to measure therapeutic response, due to treatment toxicity, poor tolerance, 
continued rapid progression of malignancy leading to complications or hospice transitions, or decision to discontinue treatment by the patient for other reasons. Of the 51 patients with measurable response, there were 27 patients who progressed, 3 patients with stable disease, 10 patients with partial response, and 11 patients with complete response. In total, 44 of the 51 patients were treated with pembolizumab, and only two patients were treated with a PD-L1 inhibitor within a clinical protocol (Supplementary Table S1). In all analyses regarding response to ICI, patients who had CR, PR, or SD with more than 6 months duration were grouped in a category referred as "responders" and were compared against patients with tumor progression referred to as "nonresponders". Amongst all 51 patients, 24 patients were categorized as responders, and 27 patients were categorized as nonresponders. Responders received an average 19.6 (range: 3-35) administrations of an ICI, while nonresponders received only $5.3(2-9)$ administrations. The average PFS was 910 days for patients with CR, 388 days for patients with PR, 237 days for the patients with SD, and 109 days for patients with PD. PFS was statistically significantly longer in responders with an average of 661.7 days (185 to 1825 days) vs. nonresponders who had an average of 109 days $(63-190$ days) $(p<0.01)$.

Of the 51 evaluable patients, there were 36 patients with available PD-L1 expression data with 19 patients defined as responders and 17 patients as nonresponders. There were no associations found between PD-L1 level and response to treatment with ICI. When compared as a continuous variable, the mean PD-L1 was 26.4 (95\% CI 9.6-43.2) in responders vs. $26.5(95 \% \mathrm{CI} 11.0-41.9)$ in nonresponders $(p=0.99)$. When compared as a three-tiered variable, the percentage of responders and nonresponders in the 3tPD-L1-(0) category was $10.5 \%$ and $11.6 \%$, respectively, and $36.8 \%$ and $41.1 \%$ in the 3tPD-L1-(20+) category $(p=0.89)$. Similarly, there were no associations identified between PD-L1 and PFS in patients treated with ICIs. PFS was 208 days for 3tPD-L1-(0), 374 days for 3tPD-L1-(1-19), and 404 days for the 3tPD-L1-(20+) category $(p=0.66)$. There was no significant correlation between PD-L1 measured as a continues variable and PFS $(p=0.62)$

There were 40 patients with available TMB data that were able to be evaluated for treatment response, with 20 patients categorized as responders and 20 patients categorized as nonresponders. There was a statistically significant association between the response to treatment with ICI and continuous TMB, with a mean TMB of 11.3 mut $/ \mathrm{Mb}(95 \%$ CI 6.6 mut/Mb-16.0 mut/Mb) in responders and 4.9 mut/Mb (95\% CI 3.4 mut/Mb-6.4 $\mathrm{mut} / \mathrm{Mb})$ in nonresponders $(p=0.01)$. Correlation of treatment response with TMB as a categorical variable demonstrated a similar correlation, with 12 responders $(60 \%$ of the total responders) and 6 nonresponders (30\% of the total responders) found within the TMB- $(6+)$ category $(p=0.056)$. Similarly, there was a statistically significant association of TMB with PFS when analyzed as a continues variable $(p=0.01)$, and statistical significance was maintained in a categorical analysis, with PFS found to be 261.7 days in the TMB-(0-5) and 538.7 in the TMB- $(6+)(p=0.04)$.

\section{Discussion}

The establishment of PD-L1 ICIs has brought about a new era in the management of patients with HNSCC. Efforts in clinical research are now focused on defining strategies to increase the efficacy of PD-L1 ICIs by identifying those who are best suited to receive these therapies. PD-L1 and TMB have been the dominant targets investigated as potential biomarkers of response to ICIs, yet, especially in HNSCC, the results remain not only scarce but frequently inconsistent.

In this single-institution retrospective analysis, the PD-L1 and TMB data of $132 \mathrm{HN}$ SCC patients (95 patients with PD-L1 data, 128 patients with TMB data, and 91 patients with both PD-L1 and TMB data) were correlated with their demographics, survival and, when appropriate, response to ICI. This study population is consistent with a standard HNSCC population in terms of age, gender, race, smoking, and HPV status (Table 1). Conventional prognostication tools held true in this analysis. Smoking, as well as advanced nodal stage were associated with worse survival in a multivariate analysis (Table 4). Both 
PD-L1 and TMB were measured with standardized, commercially available methods. To the authors' knowledge, this is one of few studies involving a comprehensive analysis of both TMB and PD-L1 in a dedicated HNSCC population, utilizing universally available, standardized measurements of each variable. Published studies present frequently conflicting results, most likely affected by variations in the utilized assays, as well as variations in the thresholds used to define results.

The median PD-L1 score in this study was $10 \%$, and $87 \%$ of patients with PD-L1 data expressed PD-L1 positivity (PD-L1 expression $\geq 1$ ). This proportion of PD-L1 positive disease is slightly higher than previous HNSCC cohorts analyzed in the literature where studies have demonstrated positivity rates between $57 \%$ and $82 \%[5,10,11]$. It was thought that earlier disease stage might account for this finding, as significantly more patients in this study who were diagnosed at an earlier stage (overall and with respect to $\mathrm{T}$ stage alone) were found to have higher PD-L1 expression (Table 2), and there was a relative surplus of early-stage patients in the study cohort in comparison to the predominance of recurrent/metastatic disease in cohorts from the literature.

PD-L1 was evaluated as both a three-tiered and two-tiered variable. The tier cutoff points of the 3tPD-L1 variable were influenced by the KEYNOTE-048 study [32]. Although the three tiered approach had the considerable advantage of distinguishing PD-L1 negative disease (3tPD-L1-(0)), an important category in the decision tree guiding therapy in the current standard first line management of metastatic/recurrent HNSCC, the distribution of the patients in the resultant groups were dissimilar. In an attempt to help offset the bias introduced by the lack of uniformity in the methods used to measure PD-L1 expression (TPS vs. CPS) and the low number of patients in the 3tPD-L1-(0) group, the decision was made to combine the lower two categories into a single group, thereby generating the two-tiered variable (2tPD-L1). Ultimately, there were more significant associations between the 3tPD-L1 variable and demographic data, thus illustrating the importance of distinguishing PD-L1 positivity from PD-L1 negativity in HNSCC.

The median TMB analyzed was 5 mut/Mb, consistent with other reports [33]. Although the split of TMB between groups was based on Foundation Medicine guideline, the groups would be similar for a threshold based on median TMB, with 70 patients in the TMB- $(<6)$ group and 58 patients in the TMB- $(6+)$ group. Similar to the reports in HNSCC $[27,28]$ and in other malignancies $[24,33,34]$, this study demonstrated that there was no significant relationship between PD-L1 and TMB, reflecting the dynamic interactions between the two variables.

This study is the first to demonstrate an association between higher PD-L1 expression and the African American race. This held true in both the 2tPD-L1 and the 3tPD-L1 analysis. Notably, the 3tPD-L1 analysis also correlated Caucasian race to lower PD-L1 in a statistically significant manner. Conversely, a higher proportion of Caucasians had high TMB-(6+) than African Americans (48.1\% vs. 23\%), although this higher proportion was not statistically significant.

A study of The Cancer Genome Atlas (TCGA) HNSCC population did not find any significant correlation between TMB and race or gender but did identify a statistically significant association between high TMB and age above 60 [34]. This finding was confirmed by other reports on the same public TCGA HNSCC database [35] as well as in another study involving 100,000 human cancer genomes in which many malignancies were considered [33]. Such correlations of TMB or PD-L1 with age were not identified in this study.

Female gender was the only category in this analysis to show a trend toward both higher PD-L1 expression (48\% of females vs. 35.3\% of males were in the 3tPD-L1-(20+) group) and higher TMB (53.8\% of females vs. $41.6 \%$ of males were in the TMB-(6+) group). This trend did not reach statistical significance possibly due to the inherent lower number of females inflicted by this type of cancer and subsequently being included in our analysis. Similar association of PD-L1 overexpression with female gender was reported by two studies in patients with oral cavity SCC [36,37], while a retrospective study of patients with 
oropharyngeal HNSCC reported no significant difference in age and gender and PD-L1 expression [38].

Smokers were found to have both a lower level of PD-L1 expression and higher TMB, compared with never-smokers or former smokers. This TMB finding is further supported by two other recent reports. One such project was dedicated to a population size similar to this study [28] and another analyzed the TCGA-HNSCC [34]. Our study additionally demonstrated an association between alcohol consumption and lower PD-L1 expression. A review of the literature revealed two studies, focused on oral cavity SCC on this topic (a meta-analysis [36] and a smaller retrospective study of 55 patients [39]) which found a similar significant relationship between low-PD-L1 with alcohol consumption.

There are no consensus data regarding HPV status in association with PD-L1 and TMB. Previous studies in HNSCC concerning patients treated with surgery and adjuvant CRT [40] and those with oropharyngeal primaries [41] reported a significant correlation of PD-L1 expression with p16 status. Conversely, another study regarding patients with oropharyngeal SCC did not identify any significant difference in PD-L1 expression between $\mathrm{HPV}$ positive and negative tumors [38]. Similarly, there are conflicting data in the literature concerning the relationship between HPV/p16 and TMB. Some studies have shown that high TMB was associated with HPV negative disease [28,42], and others have demonstrated no significant correlation between the two variables [34]. There was no significant association between HPV and PD-L1 or TMB in this study. It was noted, however, that more patients with HPV negative disease were in the 3tPD-L1-(0) group (18.5\% of patients), and no patients with 3tPD-L1-(0) had HPV positive disease. Although these findings did not reach significance, they support the theory that the relationships between PD-L1, TMB, and immune cell infiltration are more complicated and the immune pathways that assist in response are influenced by many components of the tumor microenvironment including HPV status, alcohol use, and/or tobacco use [42].

In this cohort, a trend that did not reach statistical significance suggested that BMI greater than 30 was associated with high PD-L1 and low TMB. Interestingly, reports in 976 patients with diverse tumors treated with PD-1/PD-L1 inhibitors showed that the response to treatment was significantly higher in overweight/obese patients compared to nonoverweight patients [43].

In concordance with two other reports on patients with HNSCC [34,44], this study found that TMB was associated with tumor location in a statistically significant way. The proportion of patients with laryngeal tumors was significantly increased, and oropharyngeal cancer decreased, in the TMB-(6+) group when compared to patients with any other throat tumor location. No correlation of PD-L1 with tumor location was identified.

Advanced cancer stage (I-IV) and advanced T stage (T3-T4 vs. T0-T2) were associated with low PD-L1 in this analysis. Of the 12 patients with PD-L1(0), seven patients had $\mathrm{T} 4$, and five patients had T3 tumors (Table 4). This finding is supported by a metaanalysis in patients with oral cavity SCC [36] but diverges from a study in oropharyngeal cancer [38]. Furthermore, in a review of a TCGA HNSCC population, Zhang et al. reported an association of advanced clinical stage and large tumor size with TMB rather than with PD-L1 [42]. This correlation was not identified in our study.

PD-L1 did not correlate with survival at 1 or 2 years or with OS in univariate analysis (Table 3). In the multivariate survival analysis of the 2tPD-L1 variable, 2tPD-L1-(20+) predicted worse survival comparative with 2tPD-L1-(0-19). Previous studies have found the same, including one meta-analysis and two retrospective reviews, all addressing oral cavity SCC patients $[36,37,45]$. Another meta-analysis in HNSCC and a study in oropharyngeal SCC patients reported no association of PDL1 expression with survival $[38,46]$. Conversely, three retrospective reviews of HNSCC patients reported the association of PD-L1 expression with improved OS [40,47-49].

TMB significantly correlated with OS measured from the time of diagnosis (Table 3). This significance was maintained in a Cox proportional hazards regression model when adjusted for age, tobacco use, tumor site, nodal stage at diagnosis, previous treatment 
with chemotherapy, radiation or combined chemoradiation therapy, and PD-L1 level in a multivariate analysis model (Table 4). Similar with PD-L1, the literature reports are controversial regarding TMB's association with survival. This is not surprising given the expected influences of disease characteristics, treatment, biopsy sites, and the variability in measurement techniques. Additionally, the finding that those in the higher TMB group had a better response to PD-L1 ICI in combination with the facts that a high proportion of our patient population had TMB-(6+) and were treated with PD-L1 ICI, most likely influenced survival in this study. Reports in the literature support the correlation between high TMB and improved OS, including the reports of univariate and multivariate survival of patients with oral cavity squamous cell cancer treated with surgery as their primary intervention [50]. Conversely, a multicenter retrospective study of patients treated with definitive CRT found a significant correlation of TMB with poor survival [51]. Finally, a study of 10,000 patients from TCGA with different tumors showed an association of TMB with response to IO but not with OS [25].

A total of 79 patients in this study received at least one treatment with an ICI, of which 51 patients were evaluable for treatment response. It should be noted that the percentage of responders in this study is higher than previously reported in the literature (26.5\%), with a particularly high percentage of patients with CR (13.9\%). This finding might be correlated with the fact that almost half of these patients ( 5 out of 11 patients) were treated with other therapeutic interventions that might have potentiate immune response to ICIs (palliative radiotherapy (three patients), combined palliative chemotherapy (one patient), and concurrent definitive chemoradiotherapy for a second head and neck cancer primary (one patient)) (Supplementary Table S1). One patient with metastatic HNSCC who achieved a durable CR (1875 days to date, with no recurrence) after just three administrations of a PD-1 inhibitor will be presented in a separate publication. Of the 51 patients with evaluable response, 40 patients had TMB results, and 36 patients had PD-L1 results. There was a statistically significant association between the response to treatment with ICI and continuous TMB score with a mean TMB of 11.2 in responders and 4.9 in nonresponders $(p=0.01)$. Evaluation as a categorical variable demonstrated that $66.6 \%$ of the responders and $33.3 \%$ of the nonresponders were within the high TMB $(6+)$ category $(p=0.055)$. Furthermore, TMB corelated significantly with PFS in both categorical and continuous analysis. There are other published reports supporting TMB as a possible predictor of response to ICIs. In a retrospective analysis of $126 \mathrm{HNSCC}$ patients treated with anti-PD$1 /$ PD-L1 agents, TMB was found to be significantly higher among responders (21.3 vs. $8.2 \mathrm{mut} / \mathrm{MB}, p<0.01$ ) [28]. The study of a cohort from KEYNOTE-012 sought to characterize this further and demonstrated that TMB was predictive of response to pembrolizumab in HPV negative patients but not in HPV positive patients [29]. Finally, though the role of circulating/blood TMB has yet to be defined, retrospective studies in HNSCC have a linked response to ICI with circulating/blood TMB $\geq 16 \mathrm{mut} / \mathrm{Mb}[45,47]$.

There was no association between response to treatment with ICI or PFS and PD-L1 level analyzed as a categorical ( $p=0.66$ and $p=0.89$, respectively) or continuous variable ( $p=0.62$ and $p=0.99$, respectively). In this study, PD-L1 values were measured by both TPS ( $55 \%$ of patients) and CPS ( $45 \%$ of patients), and due to the small sample size, no attempts were made to separate the analysis of PD-L1 by the reporting technique and correlate each of these distinct groups with PFS or response to treatment with ICI. It should be noted that the literature suggests that such an analysis could yield a different result; KEYNOTE-040 and -048 reported PD-L1 by CPS and demonstrated a significant correlation between PD-L1 and response to ICI $[10,52,53]$, but CHECKMATE-141 failed to show a significant correlation between tumor response to Nivolumab and PD-L1 overexpression when PD-L1 was reported by TPS [5].

In summary, this study reported significant association of high PD-L1 expression with the African American race, nonsmoking and nonalcohol use, with early clinical cancer stage and early tumor stage, and with poor survival in a multivariate analysis. No predictive value for PFS or for BOR to ICIs was identified in the PD-L1 analysis. High TMB was reported to 
be significantly associated with smoking, tumor location in the larynx, and survival in both univariate and a multivariate analysis, as well as with PFS and BOR to ICIs.

Notably, this study comprehensively analyzed both PD-L1 and TMB in a dedicated HNSCC cohort. The utilization of standardized, commercially available methodologies is another unique feature among reports in HNSCC, encouraging the reproducibility and building of a consistent database. A direct comparison between TMB and PD-L1 results was not employed due to the variation of PD-L1 reporting (TPS and CPS) triggered by the more recent approval by the FDA of CPS as a companion diagnostic. The other limitations of this study include the retrospective nature of the review and a limited sample size, especially in the analysis regarding response to ICI.

\section{Future Directions}

Furthermore, additional studies are needed to generate the necessary context and framework of standardized variables aimed to predict response of HNSCC to IO in general and to ICIs in particular. The standardization of assays is the next step in assisting with the creation of consistent results and the development of thresholds for high and low scoring groups that are both sensitive and specific in HNSCC for further predictive analysis. The recent availability of TMB as a circulating biomarker that bypasses the need for tissue procurement and allows a dynamic assessment makes it a more attractive biomarker. The association of TMB with prognosis and response to ICI presented by this study and others warrants further attention and prompts the advancement of TMB in future prospective clinical studies of ICIs, with the ultimate goal of becoming a companion diagnostic for recommendation of ICIs in HNSCC.

\section{Conclusions}

ICIs have changed the landscape of the treatment of HNSCC. Regardless, less than $20 \%$ of the treated patients benefit from these novel therapeutics, prompting urgent studies to help identify predictors of response and improve patient selection. This study has demonstrated the utility of TMB as a prognostic variable and predictive marker of response to ICI. In addition, the study pointed to the significant association of high TMB with active tobacco use and with primary tumor location in the larynx. High PD-L1 values were associated with the African American race, high T stage, high overall disease stage, non/ex-smokers, and non-/ex-drinkers. More information is needed to create a framework in which PD-L1 and TMB co-exist with other variables to predict response to ICI on an in-dividual level. Nonetheless, the existing data for each of these independent variables are promising in the world of precision oncology, and the results of the current study argue for the advancement of TMB in prospective research.

Supplementary Materials: The following are available online at https:/ /www.mdpi.com/article/ 10.3390/ cancers13225733/s1, Table S1: Characterization of Patients who Received Immunotherapy.

Author Contributions: Conceptualization, M.P., W.Z., R.B.D.J., K.M.B., C.M.F., and U.T.; methodology, M.P., R.B.D.J., K.M.B., R.T.H., T.L.J., and P.M.B.; investigation, K.M.B., J.W.L., E.G., A.A, and A.X.; formal analysis, R.B.D.J. and K.M.B.; data curation, M.P., J.W.L., A.T.F., A.A., P.M.B., and U.T.; writing-original draft preparation, J.W.L., M.P., and J.T.B.; writing—review and editing, M.P., K.M.B., J.W.L., A.T.F., E.G., J.T.B., A.X., R.T.H., T.L.J., P.M.B., C.M.F., and U.T.; visualization, K.M.B., J.W.L., A.T.F., E.G., C.J., and A.H.S.; supervision, M.P., W.Z., R.B.D.J., R.T.H., T.L.J., C.M.F., and U.T. All authors have read and agreed to the published version of the manuscript.

Funding: Biostatistical and bioinformatics services were supported by the Comprehensive Cancer Center of Wake Forest University National Cancer Institute Cancer Center Support Grant P30CA012197. Cristina M. Furdui and Mercedes Porosnicu's effort was partly supported by NIH/NCI U01 CA215848.

Institutional Review Board Statement: The study was conducted according to the guidelines of the Declaration of Helsinki and approved by the Wake Forest School of Medicine Institutional Review Board (IRB00057787). 
Informed Consent Statement: Patient consent was waived in this retrospective study, as the research involved no risk for participants, and waiving consent did not adversely affect the subjects. Furthermore, all data were completely deidentified from time of its collection from the electronic medical record.

Data Availability Statement: The datasets analyzed are available from the corresponding author upon request.

Conflicts of Interest: The authors declare no conflict of interest. No outsider funding source contributed to this research, and therefore, there was no conflict of interest from a funding source.

\section{References}

1. Ferris, R.L. Immunology and immunotherapy of head and neck cancer. J. Clin. Oncol. 2015, 33, 3293. [CrossRef]

2. Mandal, R.; Şenbabaoğlu, Y.; Desrichard, A.; Havel, J.J.; Dalin, M.G.; Riaz, N.; Lee, K.-W.; Ganly, I.; Hakimi, A.A.; Chan, T.A.; et al. The head and neck cancer immune landscape and its immunotherapeutic implications. JCI Insight 2016, 1, e89829. [CrossRef] [PubMed]

3. Perri, F.; Ionna, F.; Longo, F.; Scarpati, G.D.V.; De Angelis, C.; Ottaiano, A.; Botti, G.; Caponigro, F. Immune response against head and neck cancer: Biological mechanisms and implication on therapy. Transl. Oncol. 2020, 13, 262-274. [CrossRef]

4. Seiwert, T.Y.; Burtness, B.; Mehra, R.; Weiss, J.; Berger, R.; Eder, J.P.; Heath, K.; McClanahan, T.; Lunceford, J.; Gause, C. Safety and clinical activity of pembrolizumab for treatment of recurrent or metastatic squamous cell carcinoma of the head and neck (KEYNOTE-012): An open-label, multicentre, phase 1b trial. Lancet Oncol. 2016, 17, 956-965. [CrossRef]

5. $\quad$ Ferris, R.L.; Blumenschein Jr, G.; Fayette, J.; Guigay, J.; Colevas, A.D.; Licitra, L.; Harrington, K.; Kasper, S.; Vokes, E.E.; Even, C. Nivolumab for recurrent squamous-cell carcinoma of the head and neck. N. Engl. J. Med. 2016, 375, 1856-1867. [CrossRef]

6. Chow, L.Q.M.; Haddad, R.; Gupta, S.; Mahipal, A.; Mehra, R.; Tahara, M.; Berger, R.; Eder, J.P.; Burtness, B.; Lee, S.-H. Antitumor activity of pembrolizumab in biomarker-unselected patients with recurrent and/or metastatic head and neck squamous cell carcinoma: Results from the phase Ib KEYNOTE-012 expansion cohort. J. Clin. Oncol. 2016, 34, 3838. [CrossRef] [PubMed]

7. Soulieres, D.; Cohen, E.; Le Tourneau, C.; Dinis, J.; Licitra, L.; Ahn, M.-J.; Soria, A.; Machiels, J.-P.; Mach, N.; Mehra, R. Abstract CT115: Updated survival results of the KEYNOTE-040 study of pembrolizumab vs standard-of-care chemotherapy for recurrent or metastatic head and neck squamous cell carcinoma 2018. In Proceedings of the AACR Annual Meeting 2018, Chicago, IL, USA, 14-18 April 2018.

8. Larkins, E.; Blumenthal, G.M.; Yuan, W.; He, K.; Sridhara, R.; Subramaniam, S.; Zhao, H.; Liu, C.; Yu, J.; Goldberg, K.B. FDA approval summary: Pembrolizumab for the treatment of recurrent or metastatic head and neck squamous cell carcinoma with disease progression on or after platinum-containing chemotherapy. Oncologist 2017, 22, 873. [CrossRef]

9. Oliva, M.; Spreafico, A.; Taberna, M.; Alemany, L.; Coburn, B.; Mesia, R.; Siu, L.L. Immune biomarkers of response to immunecheckpoint inhibitors in head and neck squamous cell carcinoma. Ann. Oncol. 2019, 30, 57-67. [CrossRef]

10. Bauml, J.; Seiwert, T.Y.; Pfister, D.G. Pembrolizumab for platinum- and cetuximab-refractory head and neck cancer: Results from a single-arm, phase II study. J. Clin. Oncol. 2017, 35, 1542-1549. [CrossRef]

11. Le, X.; Ferrarotto, R.; Wise-Draper, T.; Gillison, M. Evolving Role of Immunotherapy in Recurrent Metastatic Head and Neck Cancer. J. Natl. Compr. Cancer Netw. 2020, 18, 899-906. [CrossRef]

12. Ulrich, B.C.; Guibert, N. Non-invasive assessment of tumor PD-L1 status with circulating tumor cells. Ann. Transl. Med. 2018, 6, S48. [CrossRef] [PubMed]

13. Campesato, L.F.; Barroso-Sousa, R.; Jimenez, L.; Correa, B.R.; Sabbaga, J.; Hoff, P.M.; Reis, L.F.L.; Galante, P.A.F.; Camargo, A.A. Comprehensive cancer-gene panels can be used to estimate mutational load and predict clinical benefit to PD-1 blockade in clinical practice. Oncotarget 2015, 6, 34221. [CrossRef]

14. Johnson, D.B.; Frampton, G.M.; Rioth, M.J.; Yusko, E.; Xu, Y.; Guo, X.; Ennis, R.C.; Fabrizio, D.; Chalmers, Z.R.; Greenbowe, J. Targeted next generation sequencing identifies markers of response to PD-1 blockade. Cancer Immunol. Res. 2016, 4, 959-967. [CrossRef]

15. George, T.J.; Frampton, G.M.; Sun, J.; Gowen, K.; Kennedy, M.; Greenbowe, J.R.; Schrock, A.B.; Ali, S.M.; Klempner, S.J.; Hezel, A.F. Tumor mutational burden as a potential biomarker for PD1/PD-L1 therapy in colorectal cancer. J. Clin. Oncol. 2016, $34,3587$. [CrossRef]

16. Alexandrov, L.B.; Nik-Zainal, S.; Wedge, D.C.; Aparicio, S.A.J.R.; Behjati, S.; Biankin, A.V.; Bignell, G.R.; Bolli, N.; Borg, A.; Børresen-Dale, A.-L. Signatures of mutational processes in human cancer. Nature 2013, 500, 415-421. [CrossRef] [PubMed]

17. Kowanetz, M.; Zou, W.; Shames, D.S.; Cummings, C.; Rizvi, N.; Spira, A.I.; Frampton, G.M.; Leveque, V.; Flynn, S.; Mocci, S. Tumor mutation load assessed by FoundationOne (FM1) is associated with improved efficacy of atezolizumab (atezo) in patients with advanced NSCLC. Ann. Oncol. 2016, 27, vi23. [CrossRef]

18. Rosenberg, J.E.; Hoffman-Censits, J.; Powles, T.; Van Der Heijden, M.S.; Balar, A.V.; Necchi, A.; Dawson, N.; O’Donnell, P.H.; Balmanoukian, A.; Loriot, Y. Atezolizumab in patients with locally advanced and metastatic urothelial carcinoma who have progressed following treatment with platinum-based chemotherapy: A single-arm, multicentre, phase 2 trial. Lancet 2016, 387, 1909-1920. [CrossRef] 
19. Rosenberg, J.E.; Petrylak, D.P.; Van Der Heijden, M.S.; Necchi, A.; O’Donnell, P.H.; Loriot, Y.; Retz, M.; Perez-Gracia, J.L.; Bellmunt, J.; Grivas, P. PD-L1 expression, Cancer Genome Atlas (TCGA) subtype, and mutational load as independent predictors of response to atezolizumab (atezo) in metastatic urothelial carcinoma (mUC.; IMvigor210). J. Clin. Oncol. 2016, 34, 104. [CrossRef]

20. Hellmann, M.D.; Ciuleanu, T.-E.; Pluzanski, A.; Lee, J.S.; Otterson, G.A.; Audigier-Valette, C.; Minenza, E.; Linardou, H.; Burgers, S.; Salman, P. Nivolumab plus ipilimumab in lung cancer with a high tumor mutational burden. N. Engl. J. Med. 2018, 378, 2093-2104. [CrossRef]

21. Legrand, F.A.; Gandara, D.R.; Mariathasan, S.; Powles, T.; He, X.; Zhang, W.; Jhunjhunwala, S.; Nickles, D.; Bourgon, R.; Schleifman, E.; et al. Association of High Tissue TMB and Atezolizumab Efficacy across Multiple Tumor Types. J. Clin. Oncol. 2018, 36, 12000. [CrossRef]

22. Snyder, A.; Makarov, V.; Merghoub, T.; Yuan, J.; Zaretsky, J.M.; Desrichard, A.; Walsh, L.A.; Postow, M.A.; Wong, P.; Ho, T.S. Genetic basis for clinical response to CTLA-4 blockade in melanoma. N. Engl. J. Med. 2014, 371, 2189-2199. [CrossRef] [PubMed]

23. Rizvi, N.A.; Hellmann, M.D.; Snyder, A.; Kvistborg, P.; Makarov, V.; Havel, J.J.; Lee, W.; Yuan, J.; Wong, P.; Ho, T.S. Mutational landscape determines sensitivity to PD-1 blockade in non-small cell lung cancer. Science 2015, 348, 124-128. [CrossRef]

24. Cristescu, R.; Mogg, R.; Ayers, M.; Albright, A.; Murphy, E.; Yearley, J.; Sher, X.; Liu, X.Q.; Lu, H.; Nebozhyn, M.; et al. Pan-tumor genomic biomarkers for PD-1 checkpoint blockade-based immunotherapy. Science 2018, 362, eaar3593. [CrossRef] [PubMed]

25. McGrail, D.J.; Pilié, P.G.; Rashid, N.U.; Voorwerk, L.; Slagter, M.; Kok, M.; Jonasch, E.; Khasraw, M.; Heimberger, A.B.; Lim, B.; et al. High tumor mutation burden fails to predict immune checkpoint blockade response across all cancer types. Ann. Oncol. 2021, 32, 661-672. [CrossRef] [PubMed]

26. Goodman, A.M.; Kato, S.; Bazhenova, L.; Patel, S.P.; Frampton, G.M.; Miller, V.; Stephens, P.J.; Daniels, G.A.; Kurzrock, R. Tumor mutational burden as an independent predictor of response to immunotherapy in diverse cancers. Mol. Cancer Ther. 2017, 16, 2598-2608. [CrossRef] [PubMed]

27. Seiwert, T.Y.; Haddad, R.; Bauml, J.; Weiss, J.; Pfister, D.G.; Gupta, S.; Mehra, R.; Gluck, I.; Kang, H.; Worden, F.; et al. Abstract LB-339: Biomarkers predictive of response to pembrolizumab in head and neck cancer (HNSCC). Cancer Res. 2018, 78, LB-339. [CrossRef]

28. Hanna, G.J.; Lizotte, P.; Cavanaugh, M.; Kuo, F.C.; Shivdasani, P.; Frieden, A.; Chau, N.G.; Schoenfeld, J.D.; Lorch, J.H.; Uppaluri, R. Frameshift events predict anti-PD-1/L1 response in head and neck cancer. JCI Insight 2018, 3, e98811. [CrossRef]

29. Haddad, R.I.; Seiwert, T.Y.; Chow, L.Q.M.; Gupta, S.; Weiss, J.; Gluck, I.; Eder, J.P.; Burtness, B.; Tahara, M.; Keam, B.; et al. Genomic Determinants of Response to Pembrolizumab in Head and Neck Squamous Cell Carcinoma (HNSCC). J. Clin. Oncol. 2017, 35, 6009. [CrossRef]

30. Li, W.; Wildsmith, S.; Ye, J.; Si, H.; Morsli, N.; He, P.; Shetty, J.; Yovine, A.J.; Holoweckyj, N.; Raja, R. Plasma-based tumor mutational burden (bTMB) as predictor for survival in phase III EAGLE study: Durvalumab (D) \pm tremelimumab (T) versus chemotherapy (CT) in recurrent/metastatic head and neck squamous cell carcinoma (R/M HNSCC) after platinum failure. $J$. Clin. Oncol. 2020, 38, 6511. [CrossRef]

31. Wang, Z.; Duan, J.; Cai, S.; Han, M.; Dong, H.; Zhao, J.; Zhu, B.; Wang, S.; Zhuo, M.; Sun, J.; et al. Assessment of Blood Tumor Mutational Burden as a Potential Biomarker for Immunotherapy in Patients with Non-Small Cell Lung Cancer with Use of a Next-Generation Sequencing Cancer Gene Panel. JAMA Oncol. 2019, 5, 696-702. [CrossRef]

32. Burtness, B.; Harrington, K.J.; Greil, R.; Soulières, D.; Tahara, M.; de Castro, G., Jr.; Psyrri, A.; Basté, N.; Neupane, P.; Bratland, $\AA$. Pembrolizumab alone or with chemotherapy versus cetuximab with chemotherapy for recurrent or metastatic squamous cell carcinoma of the head and neck (KEYNOTE-048): A randomised, open-label, phase 3 study. Lancet 2019, 394, 1915-1928. [CrossRef]

33. Chalmers, Z.R.; Connelly, C.F.; Fabrizio, D.; Gay, L.; Ali, S.M.; Ennis, R.; Schrock, A.; Campbell, B.; Shlien, A.; Chmielecki, J. Analysis of 100,000 human cancer genomes reveals the landscape of tumor mutational burden. Genome Med. 2017, 9, 34. [CrossRef]

34. Kumar, G.; South, A.P.; Curry, J.M.; Linnenbach, A.; Harshyne, L.A.; Ertel, A.; Fortina, P.; Luginbuhl, A. Multimodal genomic markers predict immunotherapy response in the head and neck squamous cell carcinoma. bioRxiv 2021. [CrossRef]

35. Zhang, Y.; Lin, A.; Li, Y.; Ding, W.; Meng, H.; Luo, P.; Zhang, J. Age and mutations as predictors of the response to immunotherapy in head and neck squamous cell cancer. Front. Cell Dev. Biol. 2020, 8, 608969. [CrossRef]

36. Lenouvel, D.; González-Moles, M.Á.; Ruiz-Ávila, I.; Gonzalez-Ruiz, L.; Gonzalez-Ruiz, I.; Ramos-García, P. Prognostic and clinicopathological significance of PD-L1 overexpression in oral squamous cell carcinoma: A systematic review and comprehensive meta-analysis. Oral. Oncol. 2020, 106, 104722. [CrossRef] [PubMed]

37. Lin, Y.-M.; Sung, W.-W.; Hsieh, M.-J.; Tsai, S.-C.; Lai, H.-W.; Yang, S.-M.; Shen, K.-H.; Chen, M.-K.; Lee, H.; Yeh, K.-T. High PD-L1 expression correlates with metastasis and poor prognosis in oral squamous cell carcinoma. PLoS ONE 2015, 10, e0142656. [CrossRef]

38. Kim, H.S.; Lee, J.Y.; Lim, S.H.; Park, K.; Sun, J.-M.; Ko, Y.H.; Baek, C.-H.; Son, Y.; Jeong, H.S.; Ahn, Y.C.; et al. Association Between PD-L1 and HPV Status and the Prognostic Value of PD-L1 in Oropharyngeal Squamous Cell Carcinoma. Cancer Res. Treat. 2016, 48, 527-536. [CrossRef] [PubMed]

39. Lenouvel, D.; González-Moles, M.Á.; Ruiz-Ávila, I.; Chamorro-Santos, C.; González-Ruiz, L.; González-Ruiz, I.; Ramos-García, P. Clinicopathological and prognostic significance of PD-L1 in oral cancer: A preliminary retrospective immunohistochemistry study. Oral. Dis. 2021, 27, 173-182. [CrossRef] 
40. Balermpas, P.; Rödel, F.; Krause, M.; Linge, A.; Lohaus, F.; Baumann, M.; Tinhofer, I.; Budach, V.; Sak, A.; Stuschke, M. The PD-1/PD-L1 axis and human papilloma virus in patients with head and neck cancer after adjuvant chemoradiotherapy: A multicentre study of the German Cancer Consortium Radiation Oncology Group (DKTK-ROG). Int. J. Cancer 2017, 141, 594-603. [CrossRef] [PubMed]

41. Steuer, C.E.; Griffith, C.C.; Nannapaneni, S.; Patel, M.R.; Liu, Y.; Magliocca, K.R.; El-Deiry, M.W.; Cohen, C.; Owonikoko, T.K.; Shin, D.M. A correlative analysis of PD-L1, PD-1, PD-L2, EGFR, HER2, and HER3 expression in oropharyngeal squamous cell carcinoma. Mol. Cancer Ther. 2018, 17, 710-716. [CrossRef]

42. Zhang, L.; Li, B.; Peng, Y.; Wu, F.; Li, Q.; Lin, Z.; Xie, S.; Xiao, L.; Lin, X.; Ou, Z.; et al. The prognostic value of TMB and the relationship between TMB and immune infiltration in head and neck squamous cell carcinoma: A gene expression-based study. Oral. Oncol. 2020, 110, 104943. [CrossRef]

43. Cortellini, A.; Bersanelli, M.; Buti, S.; Cannita, K.; Santini, D.; Perrone, F.; Giusti, R.; Tiseo, M.; Michiara, M.; Di Marino, P.; et al. A multicenter study of body mass index in cancer patients treated with anti-PD-1/PD-L1 immune checkpoint inhibitors: When overweight becomes favorable. J. Immunother. Cancer 2019, 7, 57. [CrossRef]

44. Cui, J.; Wang, D.; Nie, D.; Liu, W.; Sun, M.; Pei, F.; Han, F. Difference in tumor mutation burden between squamous cell carcinoma in the oral cavity and larynx. Oral. Oncol. 2021, 114, 105142. [CrossRef]

45. Maruse, Y.; Kawano, S.; Jinno, T.; Matsubara, R.; Goto, Y.; Kaneko, N.; Sakamoto, T.; Hashiguchi, Y.; Moriyama, M.; Toyoshima, T. Significant association of increased PD-L1 and PD-1 expression with nodal metastasis and a poor prognosis in oral squamous cell carcinoma. Int. J. Oral. Maxillofac. Surg. 2018, 47, 836-845. [CrossRef] [PubMed]

46. Yang, W.; Wong, M.C.M.; Thomson, P.J.; Li, K.-Y.; Su, Y. The prognostic role of PD-L1 expression for survival in head and neck squamous cell carcinoma: A systematic review and meta-analysis. Oral. Oncol. 2018, 86, 81-90. [CrossRef] [PubMed]

47. Chen, S.-W.; Li, S.-H.; Shi, D.-B.; Jiang, W.-M.; Song, M.; Yang, A.-K.; Li, Y.-D.; Bei, J.-X.; Chen, W.-K.; Zhang, Q. Expression of PD-1/PD-L1 in head and neck squamous cell carcinoma and its clinical significance. Int. J. Biol. Markers 2019, 34, 398-405. [CrossRef] [PubMed]

48. Müller, T.; Braun, M.; Dietrich, D.; Aktekin, S.; Höft, S.; Kristiansen, G.; Göke, F.; Schröck, A.; Brägelmann, J.; Held, S.A.E. PD-L1: A novel prognostic biomarker in head and neck squamous cell carcinoma. Oncotarget 2017, 8, 52889. [CrossRef]

49. Hanna, G.J.; Woo, S.-B.; Li, Y.Y.; Barletta, J.A.; Hammerman, P.S.; Lorch, J.H. Tumor PD-L1 expression is associated with improved survival and lower recurrence risk in young women with oral cavity squamous cell carcinoma. Int. J. Oral. Maxillofac. Surg. 2018, 47, 568-577. [CrossRef]

50. Moreira, A.; Poulet, A.; Masliah-Planchon, J.; Lecerf, C.; Vacher, S.; Chérif, L.L.; Dupain, C.; Marret, G.; Girard, E.; Syx, L. Prognostic value of tumor mutational burden in patients with oral cavity squamous cell carcinoma treated with upfront surgery. ESMO Open 2021, 6, 100178. [CrossRef] [PubMed]

51. Eder, T.; Hess, A.K.; Konschak, R.; Stromberger, C.; Jöhrens, K.; Fleischer, V.; Hummel, M.; Balermpas, P.; Von Der Grün, J.; Linge, A. Interference of tumour mutational burden with outcome of patients with head and neck cancer treated with definitive chemoradiation: A multicentre retrospective study of the German Cancer Consortium Radiation Oncology Group. Eur. J. Cancer 2019, 116, 67-76. [CrossRef] [PubMed]

52. Mehra, R.; Seiwert, T.Y.; Gupta, S.; Weiss, J.; Gluck, I.; Eder, J.P.; Burtness, B.; Tahara, M.; Keam, B.; Kang, H.; et al. Efficacy and safety of pembrolizumab in recurrent/metastatic head and neck squamous cell carcinoma: Pooled analyses after long-term follow-up in KEYNOTE-012. Br. J. Cancer 2018, 119, 153-159. [CrossRef] [PubMed]

53. Cohen, E.E.W.; Soulières, D.; Le Tourneau, C.; Dinis, J.; Licitra, L.; Ahn, M.-J.; Soria, A.; Machiels, J.-P.; Mach, N.; Mehra, R. Pembrolizumab versus methotrexate, docetaxel, or cetuximab for recurrent or metastatic head-and-neck squamous cell carcinoma (KEYNOTE-040): A randomised, open-label, phase 3 study. Lancet 2019, 393, 156-167. [CrossRef] 